A. Gasparyan, A. Aptroot, A. R. Burgaz, V. Otte, Z. Zakeri, V. J. Rico, E. Araujo, A. Crespo, P. K. Divakar \& H. T. Lumbsch

\title{
First inventory of lichens and lichenicolous fungi in the Khosrov Forest State Reserve, Armenia
}

\begin{abstract}
Gasparyan, A. Aptroot, A., Burgaz, A. R., Otte, V., Zakeri, Z., Rico, V. J., Araujo, E., Crespo, A., Divakar, P. K. \& Lumbsch, H. T.: First inventory of lichens and lichenicolous fungi in the Khosrov Forest State Reserve, Armenia. — Fl. Medit. 25: 105-114. 2015. — ISSN: 1120-4052 printed, 2240-4538 online.

In 2015, an international lichenological excursion to Armenia was organized by the Young Biologists Association NGO and Organization for the Phyto-Taxonomic Investigation of the Mediterranean Area. One of the main goals of this excursion was to study lichen diversity of the Khosrov Forest State Reserve. As a result of this inventory, 176 species of lichenized and lichenicolous fungi have been found in the protected area. Out of these, 49 are reported for the first time from Armenia: Acarospora versicolor, Agonimia tristicula, Anema decipiens, Arctomia fascicularis, Arthonia intexta, A. phaeophysciae, Aspicilia cf. glomerulans, A. intermutans, Bacidina arnoldiana, Bagliettoa calciseda, Bilimbia sabuletorum, Blennothallia crispa, Chrysopsora testacea, Collema subflaccidum, Diploschistes gypsaceus, Endocarpon pusillum, Gonohymenia nigritella, G. schleicheri, Gyalolechia juniperina, Immersaria iranica, Lecania rabenhorstii, Lecanora barkmaniana, L. juniperina, L. semipallida, Leprocaulon microscopicum, Llimoniella phaeophysciae, Lobothallia recedens, Peccania coralloides, Peltula euploca, Physconia thorstenii, Piccolia ochrophora, Placidium lacinulatum, Placopyrenium fuscellum, Psorotichia schaereri, Rinodina colobina, R. obnascens, Scytinium gelatinosum, S. turgidum, Solenopsora holophaea, Thermutis velutina, Toninia candida, T. squalida, Tremella phaeophysciae, Usnea lapponica, U. wasmuthii, Verrucaria dolosa, V. macrostoma, Xanthoparmelia protomatrae and X. tinctina.
\end{abstract}

Key words: biodiversity, lichenology, protected areas, new records, oak and juniper forests, South Caucasus.

\section{Introduction}

The area of the Khosrov Forest State Reserve has already been recognized as a protected area in the fourth century for hunting and conservation reasons by the Armenian king Khosrov Kotak. In 1958, the Khosrov Forest has officially been established as a State Reserve (Khanjyan 2004). The aim of this protected area is to preserve oak and juniper forest ecosystems and montane vegetation that supports numerous threatened species of 
plants and animals. The state reserve is under strict protection, corresponding to IUCN category I. The reserve area covers 29196 ha territory in the central part of the Ararat province in Southern Armenia (Fig. 1) (Anonymous 1999). It is situated between the slopes south of Geghama and north-west of Urts and north-east of the Yeranos mountain ranges, at an altitude varying between 900 and $2500 \mathrm{~m}$. The climate is dry continental with annual precipitation of 350 to $800 \mathrm{~mm}$ (Anonymous 2008).

The area is known by its exceptionally rich biodiversity and natural landscapes of frigid vegetation, open arid forests and montane steppes (Fig. 2). Currently 1849 species of vascular plants (including 24 endemic species) and 283 animal species are known from the reserve (Anonymous 2008). The semi-arid and frigid formations occur on foothills and the lower mountain belt, where dominated a vegetation of Artemisia fragrans, Salsola ericoides, S. dendroides, etc. The forest ecosystems (1400-2300 m) are generally dominated by oak trees (Quercus macranthera) and sparse juniper (Juniperus polycarpos, etc.) formations, accompanied by Fraxinus excelsior, Sorbus aucuparia, Acer, Pyrus, etc., species (Khanjyan 2004). The riverine forest vegetation is predominated by Fraxinus, Populus, Salix, etc., species. Various grasses, such as the feather grass (Stipa stenophylla, S. capil-

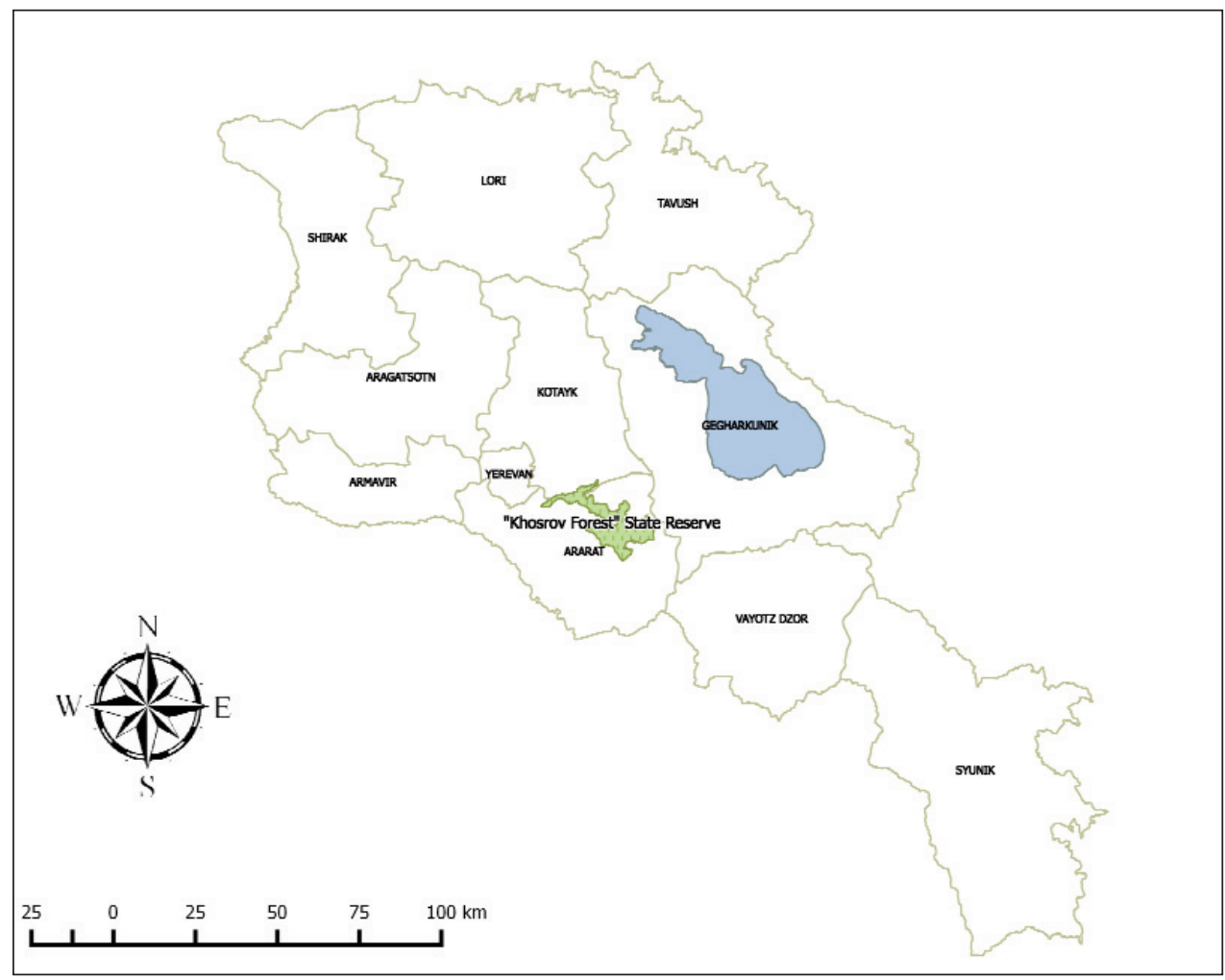

Fig. 1. The map of the Republic of Armenia including marzes (provinces), and location of the Khosrov Forest State Reserve. 


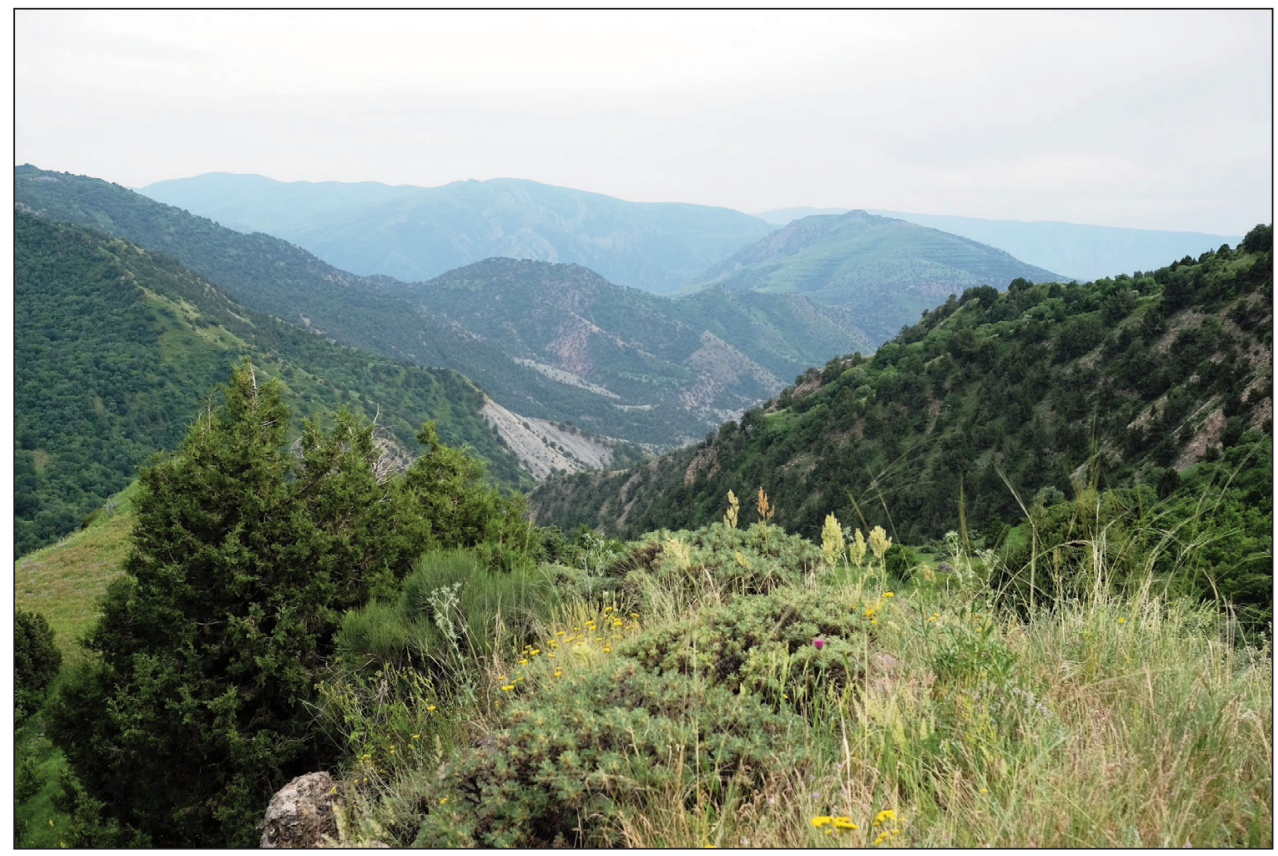

Fig. 2. Natural landscapes in the Khosrov Forest State Reserve (photo by Víctor J. Rico).

lata, etc.), tragacanth (Astragalus microcephalus and A. lagurus) motley grass steppe, etc., dominate in the montane steppes (Anonymous 2008).

Currently, 464 lichens and 2 lichenicolous fungi species are known from Armenia (Harutyunyan \& al. 2011; Gasparyan \& Sipman 2013; Gasparyan \& al. 2014). In the protected area, mainly higher plants, fungi and animals have been investigated and monitored. There are only a few previous lichen records known from the reserve. No comprehensive lichenological surveys have been carried out in the area till date, which could be important for our understanding of lichen diversity in Armenia.

This paper presents the results of the first inventory of lichens in the Khosrov Forest State Reserve, which has been carried out during an international lichenological excursion to Armenia. The excursion was organized in the frame of the OPTIMA Iter Lichenologicum initiative by the Young Biologists Association NGO (Armenia) and OPTIMA (Organization for the Phyto-Taxonomic Investigation of the Mediterranean Area).

\section{Material and methods}

During the excursion from 17 to 18 of June, 2015, a total of six localities were visited by the participants of the excursion in the Khosrov Forest State Reserve. The specimens have been collected and identified with routine methods. Secondary metabolites have been studied by thin-layer chromatography (Orange \& al. 2010). Additionally, in order to con- 
firm the identification based on morphological features, internal transcribed spacer (ITS) of some Parmeliaceae and Physciaceae specimens have been sequenced (extracts numbered and kept in MAF-Lich.) and compared with available ITS sequences in GenBank (NCBI). Voucher specimens are deposited in the herbaria ABL, B, F, GLM, MACB, MAFLich. and PAL.

\section{List of visited localities}

1.- ARMENIA: ARARAT: Vedi, Urtsadzor, Khosrov Forest State Reserve, 3959'07'N 44 ${ }^{\circ} 53^{\prime} 51^{\prime \prime} \mathrm{E}, 1390 \mathrm{~m}, 17-\mathrm{VI}-2015$, a) on Ulmus sp., b) on calcareous soil, c) on siliceous volcanic rocks, d) on limestone, e) on Juniperus sp., f) on bark, mixed forest of Quercus, Juniperus and Acer.

2.- ARMENIA: ARARAT: Vedi, Urtsadzor, Khosrov Forest State Reserve, $40^{\circ} 00^{\prime} 42^{\prime \prime} \mathrm{N}$ 44 54'41'E, 1600 m, 17-VI-2015, a) on Fraxinus excelsior, b) on Juniperus sp., c) on siliceous volcanic rocks d) on limestone, e) on aquatic siliceous rocks, f) on rocks, $\mathbf{g}$ ) on Salix sp., h) on Quercus sp., i) on Acer monspessulanum, j) on bark, mixed forest of Quercus, Juniperus and Acer.

3.- ARMENIA: ARARAT: Vedi, Urtsadzor, Khosrov Forest State Reserve, around a

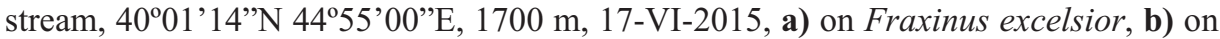
Quercus macranthera, c) on siliceous volcanic rocks, d) on soil, e) on Juniperus sp., f) on limestone, g) on bark, mixed forest of Quercus, Juniperus and Acer.

4.- ARMENIA: ARARAT: Vedi, Urtsadzor, Khosrov Forest State Reserve, top of the hill, $40^{\circ} 01^{\prime} 20^{\prime} \mathrm{N} 44^{\circ} 54^{\prime} 33^{\prime}$ 'E, $1850 \mathrm{~m}, 17-\mathrm{VI}-2015$, a) on Quercus macranthera, b) on bark, mixed forest of Quercus, Juniperus and Acer.

5.- ARMENIA: ARARAT: Goght, Khosrov Forest State Reserve, entrance from Garni,

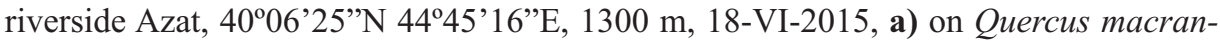
thera, b) on Populus sp., c) on quartzitic rocks, d) on siliceous volcanic rocks, e) on Fraxinus excelsior, f) on Juglans regia, g) on Cornus sp., h) on soil, i) on limestone, j) on bark, mixed forest of Quercus, Juniperus and Acer.

6.- ARMENIA: ARARAT: Vedi, Urtsadzor, Khosrov Forest State Reserve, abandoned village, $40^{\circ} 01^{\prime} 10.7^{\prime} \mathrm{N}, 44^{\circ} 54^{\prime} 46.3$ 'E, $1760 \mathrm{~m}, 17-\mathrm{VI}-2015$, a) on Malus sp.

\section{Results and discussion}

Overall, 172 species of lichenized and four species of lichenicolous fungi were found in the Khosrov Forest State Reserve. Species are listed in alphabetic order. Species names are followed by the locality or localities numbers, letter corresponding to the substrate and in case of lichenicolous fungi the host lichen species in brackets. Some specimens of Anaptychia, Melanelixia, Melanohalea, Parmelia, Parmelina and Physconia, have been sequenced, in those cases the DNA extraction number is included, in brackets, after the letter corresponding to the substrate. Forty five taxa of lichens and 4 taxa of lichenicolous fungi are new records for Armenia and marked in the list with $\left(^{*}\right), 14$ of the new records represent new records for genera in Armenia and these 
have been marked using $(* *)$. With these new records, the total number of lichens and lichenicolous fungi known from Armenia reaches 513 species, 507 lichens and 6 lichenicolous fungi.

\section{List of species}

Acarospora assimulans Vain. - 1c

Acarospora cervina (Ach.) A. Massal. - 1d

Acarospora fuscata (Nyl.) Th. Fr. $-5 \mathrm{~d}$

Acarospora insolata H. Magn. - 5d

Acarospora veronensis A. Massal. - 1c, 5d

* Acarospora versicolor Bagl. \& Car. - 3f, 5d

**Agonimia tristicula (Nyl.) Zahlbr. - 2d, 3c

Alyxoria varia (Pers.) Ertz \& Tehler - 2a

Anaptychia ciliaris (L.) A. Massal. - 2j (DNA 4965), 3g (DNA 4966)

Anaptychia roemeri Poelt $-1 \mathrm{~d}, 3 \mathrm{c}$

**Anema decipiens (A. Massal.) Forssell - 1d, 2d

*Arctomia fascicularis (L.) Otálora \& Wedin - 2a

*Arthonia intexta Almq. (in Lecidella elaeochroma apothecia) - $2 \mathrm{j}$

*Arthonia phaeophysciae Grube \& Matzer (on Phaeophyscia cf. hirsuta) - 2a, 5b

Aspicilia cinerea (L.) Körb. - 2c

Aspicilia contorta subsp. hoffmanniana $\mathrm{R}$. Sant. - 1d

Aspicilia desertorum (Kremp.) Mereschk. - 1cd, 2c, 5d

*Aspicilia cf. glomerulans (Poelt) Poelt $-5 \mathrm{~d}$

*Aspicilia intermutans (Nyl.) Arnold - 2c

Aspicilia reticulata Kremp. - 3c

Athallia pyracea (Ach.) Arup - 1ae, 2ae, 3be, 4a

* Bacidina arnoldiana V. Wirth \& Vězda - 2e

**Bagliettoa calciseda (DC.) Gueidan \& Cl. Roux - 2d

* Bilimbia sabuletorum (Schreb.) Arnold - 2d

*Blennothallia crispa (Hudson) Otálora, P.M. Jørg. \& Wedin - 3c

Calogaya biatorina (A. Massal.) Arup, Frödén \& Søchtink - 1d

Calogaya polycarpoides (J. Steiner) Arup, Frödén \& Søchtink - 3a

Calogaya pusilla (A. Massal.) Arup, Frödén \& Søchtink - 2d

Caloplaca cerina (Hedw.) Th. Fr. - 1a, 2ah

Caloplaca demissa (Körb.) Arup \& Grube - 3c

Caloplaca monacensis (Leder.) Lettau - 1ae, 2a, 3b

Candelariella antennaria Räsänen - 2e, 4a

Candelariella aurella (Hoffm.) Zahlbr. - 2d

Candelariella vitellina (Ehrh.) Müll. Arg. - 1d, 2d

**Chrysopsora testacea (Hoffm.) Choisy - 1d

Circinaria calcarea (L.) A. Nordin, S. Savić \& Tibell - 1d, 2ad, 5d

Cladonia pocillum (Ach.) O. J. Rich - 5a

Collema flaccidum (Ach.) Ach. - 2a

*Collema subflaccidum Degel. - 3b, 4a

Dermatocarpon miniatum (L.) W. Mann - 2c, 3f, 5d 
Dimelaena oreina (Ach.) Norman - 3c

*Diploschistes gypsaceus (Ach.) Zahlbr. - 2f, 3f

Diploschistes muscorum (Scop.) R. Sant. $-5 \mathrm{~h}$

Diploschistes scruposus (Schreb.) Norman - 5d

Diplotomma hedinii (H. Magn.) P. Clerc \& Cl. Roux - 2d

Enchylium tenax (Sw.) Gray - 2d

*Endocarpon pusillum Hedw. - 3f, 5d

Evernia prunastri (L.) Ach. - $3 \mathrm{~g}$

Flavoplaca flavocitrina (Nyl.) Arup, Frödén \& Søchtink - 2c

Glypholecia scabra (Pers.) Müll. Arg. - 1d

**Gonohymenia nigritella (Lettau) Henssen - 1d

* Gonohymenia schleicheri (Hepp) Henssen - 3c

Gyalolechia flavovirescens (Wulfen) Søchtink, Frödén \& Arup - 5d

* Gyalolechia juniperina (Tomin) Søchtink, Frödén \& Arup - 1b

Immersaria cupreoatra (Nyl.) Calat. \& Rambold - 1c, 2c

*Immersaria iranica Valadb., Sipman \& Rambold - 2c

Lathagrium cristatum (L.) Otálora, P.M. Jørg. \& Wedin - 1bd, 5d

Lecania cyrtella (Ach.) Th. Fr. - 3a

Lecania fuscella (Schaer.) A. Massal. - 2a, 3a

*Lecania rabenhorstii (Hepp) Arnold - 2d

Lecanora argopholis (Ach.) Ach. - 1c, 5d

*Lecanora barkmaniana Aptroot \& Herk - 3a

Lecanora bicincta Ramond - 3c

Lecanora bolcana (Pollinii) Poelt - 2c, 3c

Lecanora carpinea (L.) Vain. - 1a

Lecanora chlarotera Nyl. - 1a

Lecanora garovaglii (Körb.) Zahlbr. - 1c, 2c, 3c, 5d

*Lecanora juniperina Śliwa - 1ae, 2ab, 3be

Lecanora muralis (Schreb.) Rabenh. - 1d, 2d, 3c, 5d

Lecanora percrenata $\mathrm{H}$. Magn. - 1d, $5 \mathrm{~d}$

Lecanora rupicola (L.) Zahlbr. - 2c, 3c

*Lecanora semipallida H. Magn. - 2d, 5d

Lecanora wetmorei Śliwa - 2a, 4ab, 5abj

Lecidella elaeochroma (Ach.) M. Choisy - 2aj, 3a, 5j

Lecidella euphorea (Flörke) Hertel - 1a, 2a, 3 ag

Lecidella patavina (A. Massal.) Knoph \& Leuckert - 3c

Lepraria finkii (de Lesd.) R. C. Harris $-3 \mathrm{c}$

Lepraria nivalis J. R. Laundon $-2 \mathrm{~d}, 3 \mathrm{c}, 5 \mathrm{~d}$

**Leprocaulon microscopicum (Vill.) Gams - 3c

Leptogium saturninum (Dicks.) Nyl. - 2a, 3a

**Llimoniella phaeophysciae Diederich, Ertz \& Etayo (on Phaeophyscia cf. hirsuta) - 1a, 5b

Lobothallia alphoplaca (Wahlenb.) Hafellner - 3c, 5d

Lobothallia praeradiosa (Nyl.) Hafellner - 1cd, 2c

Lobothallia radiosa (Hoffm.) Hafellner - 3c, 5d

*Lobothallia recedens (Taylor) A. Nordin, S. Savić \& Tibell - 2c 
Megaspora rimisorediata Valadb. - 1e, 2b

Melanelixia glabra (Schaer.) O. Blanco, A. Crespo, Divakar, Essl., D. Hawksw. \& Lumbsch - 1af (DNA 5053), 2aij (DNA 5055), 3abeg (DNA 5054), $5 \mathrm{~g}$

Melanelixia subargentifera (Nyl.) O. Blanco, A. Crespo, Divakar, Essl., D. Hawksw. \& Lumbsch - 1af (DNA 5065, 5067), 2abij (DNA 5068, 5070), 3aeg, 4ab (DNA 5069), 5fj (DNA 5063)

Melanohalea elegantula (Zahlbr.) O. Blanco, A. Crespo, Divakar, Essl., D. Hawksw. \& Lumbsch - 2j, 3g (DNA 5170), 4ab (DNA 5059)

Melanohalea exasperata (De Not.) O. Blanco, A. Crespo, Divakar, Essl., D. Hawksw. \& Lumbsch - 1f, 2agi, 4a, 6a

Parmelia sulcata Taylor - 3g (DNA 5003, 5004)

Parmelina tiliacea (Hoffm.) Hale - 2acj (DNA 4961, 4962, 4963, 4988, 4989, 4990, 4991, 5002), $3 \mathrm{~g}$ (DNA 4992, 4993), 4b (DNA 4994, 4995, 4996), 6a

**Peccania coralloides (A. Massal.) A. Massal. - 3c

Peltigera canina (L.) Willd. - 2c

Peltigera elisabethae Gyeln. - 3cd

Peltigera ponojensis Gyeln. - 2c

Peltigera praetextata (Flörke) Vain. - 2c

Peltigera rufescens (Weiss) Humb. - 3cd

**Peltula euploca (Ach.) Ozenda \& Clauzade - 5d

Phaeophyscia ciliata (Hoffm.) Moberg $-2 \mathrm{~g}$

Phaeophyscia cf. hirsuta (Mereschk.) Essl. - 1ae, 2ab, 5b

Phaeophyscia nigricans (Flörke) Moberg - 1ae, 3b, 5a, 6a

Phaeophyscia orbicularis (Neck.) Moberg - 1a, 2aefi, 3a, 4ab, 5aef; 6a

Phaeophyscia sciastra (Ach.) Moberg - 2f, 3c

Physcia adscendens (Fr.) H. Olivier - 1a, 2ef, 3, 3a, 4b, 5ag

Physcia aipolia (Humb.) Fürnr. - 1af, 2aej, 3ag

Physcia biziana (A. Massal.) Zahlbr. - 1af, 2ai, 4a, 5ae

Physcia caesia (Hoffm.) Fürnr. - 3c

Physcia dimidiata (Arnold) Nyl. - 1ad, 2abf, 3e, 4a, 5f

Physcia dubia (Hoffm.) Lettau - 2c

Physcia stellaris (L.) Nyl. - 1af

Physcia tenella (Scop.) DC. - 1f, $2 \mathrm{f}$

Physconia detersa (Nyl.) Poelt - 1f, 3 af (DNA 5009)

Physconia distorta (With.) J. R. Laundon - 1a, 2d, 3ab, 5bf

Physconia enteroxantha (Nyl.) Poelt - 2i, 3bc, $5 \mathrm{~g}$

Physconia grisea (Lam.) Poelt - 2a

Physconia perisidiosa (Erichsen) Moberg - 2a, 3bg, 4a, 5j (DNA 4973)

*Physconia thorstenii A. Crespo \& Divakar - 1f (DNA 4972, 5010), 2j

**Piccolia ochrophora (Nyl.) Hafellner - 1a

*Placidium lacinulatum (Ach.) Breuss - $3 \mathrm{f}$

Placidium rufescens (Ach.) A. Massal. - 1d, 3cd

Placocarpus schaereri (Fr.) Breuss - 1d, 5d

* Placopyrenium fuscellum (Turner) Gueidan \& Cl. Roux - 2d

Placynthium nigrum (Huds.) Gray - 2d, 3c 
Pleurosticta acetabulum (Neck.) Elix \& Lumbsch - 2a

**Psorotichia schaereri (A. Massal.) Arnold - 1d

Punctelia borreri (Sm.) Krog - $1 \mathrm{f}$

Pyrenodesmia variabilis (Pers.) A. Massal. - 1bc, 2d, 5d

Ramalina farinacea (L.) Ach. - 3a

Ramalina pollinaria (Westr.) Ach. - $3 \mathrm{~g}$

Rhizocarpon disporum (Hepp) Müll. Arg. - 2c, 3c

Rhizocarpon geographicum (L.) DC. - 3c

Rhizoplaca chrysoleuca (Sm.) Zopf - 3c

Rhizoplaca melanophthalma (DC.) Leuckert - 1c

Rhizoplaca peltata (Ramond) Leuckert \& Poelt - 1c, 2c, 5d

* Rinodina colobina (Ach.) Th. Fr. - 1e, 2a

Rinodina immersa (Körb.) J. Steiner - 2cd

*Rinodina obnascens (Nyl.) H. Olivier - 2c

Rinodina pyrina (Ach.) Arnold - 3e

Romjularia lurida (Ach.) Timdal - 2d

Rusavskia elegans (Link) S.Y. Kondr. \& Kärnefelt - 5d

Sarcogyne regularis Körb. $-2 \mathrm{~d}$

*Scytinium gelatinosum (With.) Otálora, P.M. Jørg. \& Wedin - 2adf, 3ac, 5d

Scytinium lichenoides (L.) Otálora, P.M. Jørg. \& Wedin - 2afj, 3e

*Scytinium turgidum (Ach.) Otálora, P.M. Jørg. \&Wedin - 3c

**Solenopsora holophaea (Mont.) Samp. - 2d, 3c

Squamarina cartilaginea (With.) P. James - 2c

Staurothele areolata (Ach.) Lettau - 3c

Staurothele fuscocuprea (Nyl.) Zschacke - 2d

Tephromela atra (Huds.) Hafellner - 5a

**Thermutis velutina (Ach.) Flot. $-1 \mathrm{c}, 1 \mathrm{~d}, 2 \mathrm{c}$

Thyrea confusa Henssen - 1d, 2d

* Toninia candida (Weber) Th. Fr. - 2d

Toninia cinereovirens (Schaer.) A. Massal. - 1d (Fig. 3)

Toninia sedifolia (Scop.) Timdal - 3d, 5d

*Toninia squalida (Ach.) A. Massal. - 3c

**Tremella phaeophysciae Diederich \& M. S. Christ. (on Pheophyscia orbicularis) - 4b

*Usnea lapponica Vain. - 3g

Usnea substerilis Motyka - 3g

*Usnea wasmuthii Räsänen - 3g

*Verrucaria dolosa Hepp - 2e

Verrucaria hochstetteri Fr.-2d

*Verrucaria macrostoma DC. - 1d

Verrucaria muralis Ach. - 2d

Verrucaria nigrescens Pers. - 2de, 5d

Xanthocarpia lactea (A. Massal.) A. Massal. - 2d

Xanthomendoza fulva (Hoffm.) Søchting, Kärnefelt \& S.Y. Kondr. - 5b

Xanthomendoza ulophyllodes (Räsänen) Søchting, Kärnefelt \& S.Y. Kondr. - 2a, 3b, 4a, 5b

Xanthoparmelia conspersa (Ach.) Hale - 5d 


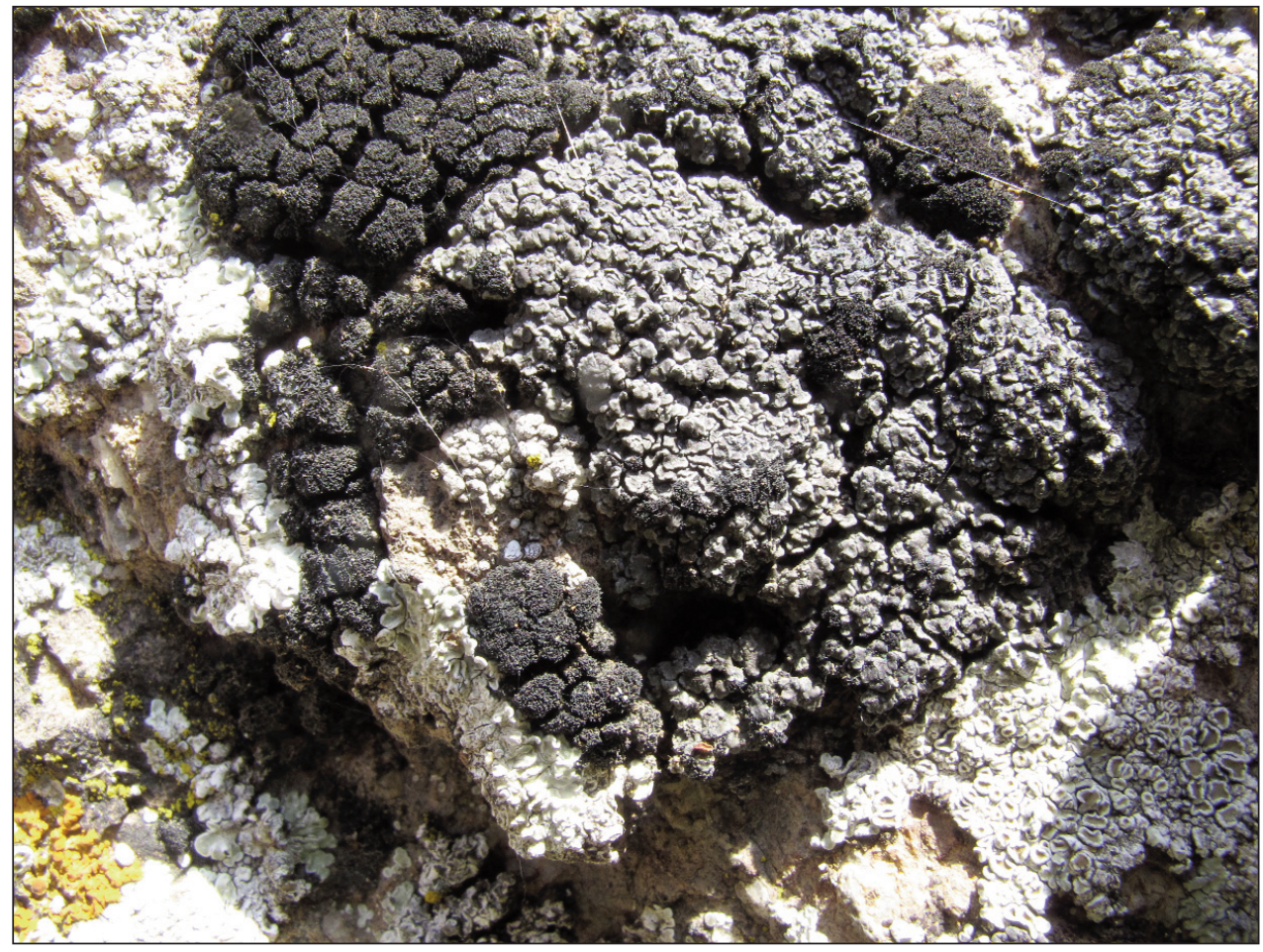

Fig. 3. Toninia cinereovirens and Thermutis velutina on limestone in the Khosrov Forest State Reserve (photo by Maaike Vervoort).

Xanthoparmelia loxodes (Nyl.) O. Blanco, A. Crespo, Elix, D. Hawksw. \& Lumbsch - 3c * Xanthoparmelia protomatrae (Gyeln.) Hale - 5d

Xanthoparmelia pulla (Ach.) O. Blanco, A. Crespo, Elix, D. Hawksw. \& Lumbsch - 1c, $2 \mathrm{c}, 3 \mathrm{c}, 5 \mathrm{dh}$

Xanthoparmelia somloensis (Gyeln.) Hale - 3c, 5dh

*Xanthoparmelia tinctina (Maheu \& A. Gillet) Hale - 1c, 2c, 3cd, 5d

Xanthoparmelia verruculifera (Nyl.) O. Blanco, A. Crespo, Elix, D. Hawksw. \& Lumbsch - 5d Xanthoria parietina (L.) Th. Fr. - 5a, 5f, 5g

\section{Acknowledgments}

The authors would like to express their gratitude to the staff of the Khosrov Forest State Reserve for kind support during field work and to the Ministry of Nature Protection for permission to collect the specimens. We are also very thankful to staff and volunteers of the Young Biologists Association NGO, especially Hripsime Atoyan, Vanuhi Hambardzumyan and Maria Antonosyan for field assistance during the excursion. The first author is especially grateful to Drs Harrie J. Sipman and Jan Vondrak for support in the identification of some specimens, as well as acknowledges financial support from DAAD (German Academic Exchange Service) and the project "Developing Tools for 
Conserving the Plant Diversity of the Transcaucasus" financed by the Volkswagen Foundation. AC, VJR, PKD and EA, acknowledges financial support from the Spanish Ministerio de Economía y Competitividad projects CGL2011-25003 and CGL2013-42498-P. ARB acknowledges financial support to Ministry of Economy and Competitiveness, Spain, project CGL2013-41839-P. We are also thankful to Maaike Vervoort for permission to publish the photograph in Fig. 3.

\section{References}

Anonymous (Ministry of Nature Protection) 1999: First National Report to The Convention on Biological Diversity (incorporating a country study on the biodiversity of Armenia). Yerevan.

- 2008: "Khosrov Forest" State Reserve management plan 2010-2014. - Yerevan (in Armenian).

Gasparyan, A. \& Sipman, H. J. M. 2013: New lichen records from Armenia. - Mycotaxon 123: 491492. doi: $10.5248 / 123.491$

_ , _ \& Brackel, W. von. 2014: A contribution to the lichen-forming and lichenicolous fungi flora of Armenia. - Willdenowia 44: 263-267. doi: 10.3372/wi.44.44208

Harutyunyan, S., Wiesmair, B. \& Mayrhofer, H. 2011: Catalogue of the lichenized fungi in Armenia. - Herzogia 24: 265-296. doi: 10.13158/heia.24.2.2011.265

Khanjyan, N. 2004: Specially protected nature areas of Armenia. - Yerevan.

Orange, A., James, P. W. \& White F. J. 2010: Microchemical methods for the identification of lichens, 2. - London.

Addresses of the authors:

Arsen Gasparyan ${ }^{1}$, André Aptroot ${ }^{2}$, Ana Rosa Burgaz ${ }^{3}$, Volker Otte ${ }^{5}$, Zakieh Zakeri ${ }^{5}$, Víctor J. Rico ${ }^{4}$, Elena Araujo ${ }^{4}$, Ana Crespo ${ }^{4}$, Pradeep K. Divakar ${ }^{4}$, H.Thorsten Lumbsch ${ }^{6}$, ${ }^{1}$ Botanischer Garten und Botanisches Museum Berlin-Dahlem, Freie Universität Berlin, Königin-Luise-Str. 6-8, 14195 Berlin, Germany. E-mail: a.gasparyan@bgbm.org

${ }^{2}$ ABL Herbarium, Gerrit van der Veenstraat 107, NL-3762 XK Soest, The Netherlands.

${ }^{3}$ Departamento de Biología Vegetal I, Faculty of Biology, calle José Antonio Novais 2, Universidad Complutense, 28040-Madrid, Spain.

${ }^{4}$ Departamento de Biología Vegetal II, Facultad de Farmacia, Universidad Complutense, Plaza de Ramón y Cajal s/n, 28040 Madrid, Spain.

${ }^{5}$ Senckenberg Museum of Natural History, Am Museum 1, 02826 Görlitz, Germany. ${ }^{6}$ Science \& Education, The Field Museum, Chicago, Illinois, USA. 